\title{
Les formations herbacées de Corse I - Élaboration d'une typologie mésologique et floristique
}

\author{
F Volaire ${ }^{1 *}$, M Godron ${ }^{2}$, F Lelièvre ${ }^{3}$ \\ 1 Station de recherches agronomiques, INRA, 20230 San Giuliano; \\ 2 Institut de botanique, 163, rue Auguste-Broussonnet, 34000 Montpellier ; \\ 3 Station d'amélioration des plantes, INRA, Domaine de Melgueil, chemin de Mézouls, 34130 Mauguio, France
}

(Reçu le 28 septembre 1989 ; accepté le 11 décembre 1989)

\begin{abstract}
Résumé - Les formations herbacées spontanées de Corse sont, malgré leur caractère résiduel, à la base de l'alimentation des troupeaux en libre parcours. L'étude de ces formations est menée sur la base d'un échantillonnage stratifié régional : 147 stations situées à moins de $1000 \mathrm{~m}$ d'altitude sont analysées. Une approche phyto-écologique révèle que les conditions hydriques édaphiques ainsi que le mode de gestion anthropique et l'intensité de pâturage sont les facteurs analytiques les plus efficaces sur la différenciation floristique des couverts. Ils permettent de construire une typologie qui met en évidence 7 types de milieux. Ceux-ci sont caractérisés par leur cortège floristique différentiel, leurs espèces dominantes indicatrices et leur répartition géographique.
\end{abstract}

formation herbacée / typologie / caractéristique du milieu / biogéographie

Summary - The natural herbaceous grasslands of Corsica.I - Construction of a typology by the characterization of both the environmental factors and the flora of the types. The natural herbaceous grasslands of Corsica provide, in spite of their scarceness, the basic feeding for herds. The study of these grasslands is based on a regional and stratified sampling: 147 grasslands (under an altitude of $1000 \mathrm{~m}$ ) are analysed. From the total of 364 species, $20.6 \%$ are legumes and $15.4 \%$ are grasses. The flora is rather rich in perennial herbaceous species (37.4\%). Data are analyzed by factorial analysis of correspondance which shows that the moisture conditions, the type of management and the intensity of grazing are the main analytical factors of differenciation of the flora (table 1). These factors are used to build a typology with 7 types (table II). The differential flora and the indicatory dominant species of every type are described (tables III and IV):

- type 1 (dry conditions, no management, under-grazing): Dactylis glomerata is the main indicatory dominant species (average covering $=30 \%$ );

- type 2 (dry conditions, no management, over-grazing): Medicago minima and Poa bulbosa (average covering $=19 \%$ ) are the main dominant species;

- type 3 (dry conditions, places regularly burnt): Anthoxanthum odoratum and Brachypodium ramosum (average covering $=29 \%$ ) are the most characteristic species;

- type 4 (average conditions of humidity, over-grazing): Lolium perenne (average covering = 20\%), Poa annua and

Trifolium nigrescens are very characteristic of this type;

- type 5 (average conditions of humidity, mowing): Vulpia myuros (average covering = 25\%) is the main species;

- type 6 (average conditions of humidity, fertilisation, good management of the stocking rate): the main species are Trifolium repens, Poa pratensis (average covering $=17 \%$ ) and Dactylis glomerata (average covering $=20 \%$ );

- type 7 (wet conditions): Trifolium fragiferum (average covering $=20.5 \%$ ) is the most characteristic species of this type.

The geographical distribution of the 7 types, in the different corsican regions, is described (table $V$ ).

natural grassland / typology / environmental characterization / biogeography 


\section{INTRODUCTION}

Comme nombre de régions méridionales françaises, la Corse a vu son paysage rural affecté par de profondes mutations. Au XIX ${ }^{\mathrm{e}}$ siècle, une économie agro-pastorale permettait l'autosubsistance d'une forte population (Pernet et Lenclud, 1977). Dans la première moitié du $x x^{\theta}$ siècle, un exode rural massif lié à une déprise agricole importante a favorisé l'essor de l'élevage qui est actuellement la principale activité économique dans l'intérieur de l'île (Simi, 1981). Cet élevage (bovins, ovins laitiers et caprins) est basé sur l'exploitation extensive d'un espace non limitant. Les parcours sont ainsi soumis à de faibles charges animales et ils se dégradent par le développement des espèces arbustives (maquis), et par la pratique de fréquentes mises à feu, ce qui induit une disponibilité fourragère insuffisante au regard des besoins du cheptel (Vallerand, 1985).

Dans ce contexte, nous avions analysé en 1984 , le cas de 3 systèmes d'élevage de HauteCorse. Les suivis de troupeaux ovins en libre parcours ont mis en évidence le rôle majeur des formations à dominante herbacée présentes sur le territoire : celles-ci représentent moins de 5\% de la superficie des parcours, alors que les ovins y prélèvent 50 à $80 \%$ de leur ration suivant les saisons (Volaire, 1985). Constatant l'importance de ces formations pour l'alimentation animale, nous avons cherché à réaliser, à l'échelle régionale, une typologie phyto-écologique de ces milieux. II s'agit donc d'une phase d'inventaire et de reconnaissance nécessaire pour apprécier l'importance relative des phénomènes en jeu (Lepart, 1984), et pour appuyer d'ultérieures expérimentations agronomiques en vue d'améliorer la gestion de cette ressource.

\section{MÉTHODES}

Nous avons étudié 147 stations écologiques de formations herbacées (où le recouvrement en ligneux est inférieur à 10\%) selon un échantillonnage stratifié sur la base de 2 variables fondamentales pour lesquelles des cartes étaient disponibles : l'altitude (de 0 à $1000 \mathrm{~m}$ ) et la nature de la roche mère. A l'intérieur des strates définies par la combinaison de ces 2 facteurs, nous avons également cherché à échantillonner la plus grande gamme possible de modes de gestion et de conditions hydriques. Sur chaque site, a été réalisé, aux printemps 1985 et 1986, un relevé phyto-écologique complet selon les méthodes de Godron et al (1968) ; c'est-à-dire que sont pris en compte : la liste exhaustive des espèces recensées sur $32 \mathrm{~m}^{2}$ avec une estimation des contributions spécifiques par la méthode des points quadrats (Daget et Poissonet, 1971) et 15 variables mésologiques énumérées dans le tableau I. Notons que toutes ces variables ont été mesurées ou estimées sur le terrain, par l'observation d'indices in situ, et par des enquêtes auprès des éleveurs quand cela était possible.

Les traitements des données visent à établir les relations existant entre descripteurs du milieu et caractéristiques floristiques. lls relèvent de 2 approches :

- l'analyse multivariée, notamment l'analyse factorielle des correspondances (AFC) permettant d'obtenir, en particulier, une image synthétique des relations entre espèces et descripteurs (Benzecri, 1973);

- l'analyse de l'information donnée par les profils écologiques, qui permet de calculer le degré d'interrelation (information) existant entre les espèces et les modalités des descripteurs écologiques (Daget et Godron, 1982). Un profil écologique est défini comme une suite de fréquences ordonnées selon les modalités d'un descripteur. Nous utilisons le profil indicé qui indique, pour chaque modalité d'une variable, la probabilité de la fréquence observée pour une espèce, par rapport à

Tableau I. Contributions absolues (CA) et contributions absolues corrigées (CAC) des variables aux 4 premiers axes de l'AFC “espèces-descripteurs" (CAC $=\mathrm{CA} /(\mathrm{N}-1)^{1 / 2}$ avec $\mathrm{N}=$ nombre de classes du descripteur $)$.

\begin{tabular}{|c|c|c|c|c|}
\hline Descripteurs & CA total & rang & $C A C$ total & rang \\
\hline Région & 844 & 1 & 218,0 & 2 \\
\hline Latitude & 564 & 2 & 252,9 & 1 \\
\hline Conditions hydriques du sol & 322 & $\overline{3}$ & 131,9 & 5 \\
\hline Profondeur de sol meuble & 268 & 4 & 109,8 & 9 \\
\hline Longitude & 261 & 5 & 130,5 & 6 \\
\hline Intensité de pâturage & 232 & 6 & 134,1 & 3 \\
\hline Artificialisation & 230 & 7 & 132,9 & 4 \\
\hline Altitude & 229 & 8 & 114,5 & 8 \\
\hline Roche mère & 206 & 9 & 119,0 & 7 \\
\hline Topographie & 201 & 10 & 82,4 & 11 \\
\hline Recouvrement végétation & 167 & 11 & 96,5 & 10 \\
\hline pH premier horizon & 135 & 12 & 55,3 & 14 \\
\hline Texture premier horizon & 130 & 13 & 53,3 & 15 \\
\hline Pente & 126 & 14 & 72,8 & 12 \\
\hline Exposition & 106 & 15 & 61,3 & 13 \\
\hline
\end{tabular}


la fréquence attendue en cas de distribution aléatoire (Gauthier et al, 1977). L'intensité de la liaison entre chaque espèce et chaque classe de variable est symbolisée par,,++++++ et,,------ (tableaux III et IV) selon qu'elle est respectivement plus ou moins forte qu'attendue avec des probabilités de $99,9 \%$, $99,0 \%$ et $95,0 \%$. Un blanc symbolise un test irréalisable du fait d'un échantillonnage insuffisant.

\section{RÉSULTATS ET DISCUSSION}

\section{La diversité floristique}

Les 364 espèces recensées appartiennent à 201 genres et 45 familles et elles comprennent : $20,6 \%$ de Légumineuses, $15,4 \%$ de Graminées et $13,7 \%$ de Composées. Ces 3 familles rassemblent environ $50 \%$ des espèces, comme dans certains pâturages sous climat méditerranéen du sud-ouest de l'Espagne, de Californie ou du Chili (Maranon-Arana, 1985). En revanche, avec $48 \%$ de Thérophytes et $37,4 \%$ d'Hémicryptophytes, la flore corse apparaît plus riche en espèces herbacées pérennes que celle des dehesas d'Andalousie, où elles représentent moins de $5 \%$ de la flore selon Vacher (1984) ou que celle des pelouses d'Isräel et du Chili, qui en comportent environ $30 \%$ d'après Maranon-Arana (op cit). Ceci peut s'expliquer d'une part, par l'étagement altitudinal de nos relevés (jusqu'à $1000 \mathrm{~m}$ ), ce qui introduit un ensemble de taxons liés à l'étage supraméditerranéen et, d'autre part, par la proximité de la mer qui atténue l'aridité estivale. La qualité du fonds pastoral de ce massif cristallin (traduite en particulier par la richesse en espèces herbacées pérennes) est une caractéristique déjà évoquée par Étienne (1977) et Joffre et Casanova (1983).

\section{Identification des descripteurs mésologiques efficaces}

Notre objectif est de hiérarchiser les descripteurs en fonction de leur sélectivité par rapport à la flore régionale, c'est-à-dire en fonction de leur efficacité. Une AFC est réalisée sur la matrice qui comporte 204 espèces (présentes au moins dans 3 relevés), et les 89 classes totalisées par les 15 variables mésologiques. II s'agit donc d'un tableau possédant 204 lignes et 89 colonnes, avec des données en présence-absence. Les 4 premiers axes rendent compte de $42,2 \%$ de l'inertie du nuage. Le tableau I propose 2 classements des variables : par rang du total de leurs contributions absolues (CA) et par rang du total de leurs contributions absolues corrigées (CAC) qui sont les $C A$ pondérées par le nombre de classes de chaque variable (Roux, comm pers).
Les deux ordinations convergent. On retiendra de la seconde, qui présente des valeurs comparables, que :

- la région et la latitude sont prépondérantes ;

- l'intensité de pâturage et le mode de gestion par l'homme (artificialisation), puis les conditions hydriques, présentent de fortes contributions ;

- la roche mère et l'altitude se détachent ensuite ;

- les caractéristiques édaphiques ( $\mathrm{pH}$ et texture) présentent les contributions les plus faibles.

Les variables latitude et longitude sont redondantes de la variable région qui apparaît de plus, par des tests de $\chi^{2}$, liée de façon très hautement significative aux conditions hydriques et à l'artificialisation (mode d'action anthropique), et de façon hautement significative à l'intensité de pâturage. Le descripteur région est donc synthétique puisqu'il intègre toute une série de liaisons à la fois entre les descripteurs mésologiques et la situation géographique et entre les descripteurs eux-mêmes. Ces résultats sont en accord avec ceux de Lepart (1984) et de Fournet et Monestiez (1987).

Les 3 facteurs analytiques les plus efficaces sur la différenciation floristique des formations sont donc : la nature des conditions hydriques, ce qui est un résultat fréquent dans les études prairiales (Gückert et Bonischot, 1984 ; Hopkins, 1986 ; Bobe, 1987) ; le mode d'action anthropique et l'intensité de pâturage qui déterminent classiquement des cortèges floristiques spécifiques (Delpech, 1975 ; Balent et Duru, 1984).

\section{Élaboration d'une typologie}

Notre typologie vise à délimiter les principaux groupements végétaux herbacés présents en Corse entre 0 et $1000 \mathrm{~m}$ d'altitude. La méthode consiste d'abord à construire des groupes de relevés homogènes sur des critères mésologiques, puis à dégager les caractères différentiels des groupes pour en vérifier la cohérence floristique; celle-ci devra être suffisante pour pouvoir assimiler un groupe de relevés à un type.

\section{Construction des groupes de relevés}

Les 3 facteurs mésologiques analytiques définis antérieurement comme étant les plus efficaces sont utilisés pour constituer un tableau à 3 entrées où sont classés les 147 relevés. Après regroupement de certaines classes des variables et élimination des combinaisons représentées par moins de 4 relevés, nous distinguons 8 groupes qui, au total, englobent 134 relevés, soit $91 \%$ des stations (tableau II). 
Tableau II. Regroupement des relevés en 8 groupes en fonction des 3 critères mésologiques principaux.

\begin{tabular}{lccccccccc}
\hline Conditions hydriques & sèches & sèches & sèches & saines & saines & saines & saines & humides \\
Artificialisation & nulle & nulle & feu & nulle & nulle & fauche & fertili & variable \\
Intensité de pâturage & faible & forte & faible & faible & forte & faible & correcte & variable & \\
Groupe & 1 & 2 & 3 & 8 & 4 & 5 & 6 & 7 & Total \\
Nombre de relevés & 10 & 14 & 9 & 24 & 33 & 19 & 15 & 10 & 134 \\
$\%$ de relevés & 7,5 & 10,4 & 6,7 & 17,9 & 24,6 & 14,2 & 11,2 & 7,5 & 100 \\
\hline
\end{tabular}

\section{Vérification de la cohérence floristique des groupes de relevés}

Ozenda (1964) rappelle qu'un groupement végétal se reconnaît souvent à des espèces dominantes qui forment l'essentiel de sa masse végétale, mais aussi à des espèces plus discrètes mais caractéristiques du milieu. La détermination de ces espèces nommées ici «différentielles» est basée sur l'analyse de l'information mutuelle existant entre les espèces et la variable synthétique appelée "groupe", créée à cet effet et qui comporte 8 modalités.

\section{Espèces différentielles discrètes}

Le tableau III donne le classement des espèces présentant les plus fortes informations. Ces taxons sont triés sur les signes positifs de leurs profils indicés.

\section{Espèces différentielles dominantes}

Le tableau IV donne le même type de classement mais réalisé sur les espèces dominantes (espèces faisant partie des 3 taxons possédant les plus fortes "contributions spécifiques" dans chaque station).

Nous allons discuter les résultats présentés dans ces 2 tableaux, en les mettant notamment en rapport avec les connaissances phytosociologiques disponibles sur les formations herbacées méditerranéennes.

Les groupes 1 et 2 , en conditions sèches, correspondent à des communautés calcifuges, thérophytiques, à caractère xérophytique et de distribution essentiellement méditerranéenne. En effet, si Lotus cytisoïdes et Paronychia argentea sont les espèces les plus "fidèles", on note que parmi les espèces qui sont particulièrement fréquentes dans ces 2 groupes, certaines appartiennent à la classe des Tuberiaretea guttatae (Loisel, 1976; Rivas Martinez, 1977; Gehu, 1977 ; Allier et Lacoste, 1981), ce sont : Briza maxima, Teesdalia coronopifolia, Ornithopus compressus, Tuberaria guttata, Paronychia argentea, Trifolium tomentosum, Sedum caespitosum et Vulpia myuros.

Le groupe 1 (sous-pâturage, absence de gestion) se distingue par la forte dominance de Dactylis glomerata qui en fait un faciès particulier. La contribution spécifique de cette espèce, lorsqu'elle apparaît dominante dans les relevés, est de $30 \%$ en moyenne. Les taxons codominants de Dactylis glomerata peuvent être : Trifolium cherleri, Thrincia tuberosa et Cynodon dactylon. Dactylis glomerata est aussi significativement dominant dans le groupe 6 (milieux frais et fertilisés) ; 2 sous-espèces ou 2 écotypes sont probablement en cause.

Le groupe 2 (sur-pâturage, absence de gestion) correspond à des communautés pionnières liées à des biotopes très pâturés. En effet, la présence de Poa bulbosa comme espèce dominante caractéristique, conduit à rapprocher ce groupe de l'ordre des Poetalia bulbosae. Dans les relevés où Poa bulbosa domine, ce taxon présente une contribution spécifique moyenne (CSm) de $19 \%$. Vulpia myuros $(\mathrm{CSm}=25 \%)$, Plantago lanceolata et Plantago coronopus ( $\mathrm{CSm}=17,5 \%$ ) (résultats non présentés dans les tableaux), Trifolium subterraneum $(\mathrm{CSm}=17 \%)$ et Medicago minima $(\mathrm{CSm}=11 \%)$ sont les espèces fréquemment codominantes de $\mathrm{Poa}$ bulbosa.

Le groupe 3 correspond à l'ensemble des stations incendiées; Daphne gnidium, Euphorbia peplus et Vicia disperma sont parmi les espèces les plus «fidèles" de ce groupe. Certaines espèces de la classe des Tuberiaretea guttatae y sont assez fréquentes : Aira caryophyllea et Lathyrus angulatus. II semble que la présence de jeunes pousses de ligneux, tels Quercus ilex, Cistus monspeliensis, Santolina chamaecyparissus, ainsi que celle de Pulicaria odora, Daphne gnidium et Carlina corymbosa, dénote que nous avons là un groupement de passage vers les cistaies (Bonin et al, 1983). II s'agirait donc d'une 
Tableau III. Ordination des espèces différentielles de la variable "groupe», classées sur les signes positifs de leur profil écologique indicé (inf mut = information mutuelle entre une espèce et la variable "groupe»). Détail des notations voir texte chapitre méthode.

Espèces différentielles

$\begin{array}{llllllll}1 & 2 & 3 & 4 & 5 & 6 & 7\end{array}$

8 Inf mut

Du groupe 1

Cynodon dactylon (L) Pers

Briza maxima $\mathrm{L}$

Lotus cytisoides (L)

Reichardia picroides (L) Roth

Scilla autumnalis L

Teesdalia coronopifolia (JP Bergeret) Thell

Vicia atropurpurea Desf

Thrincia tuberosa $\mathrm{L}$

\begin{tabular}{|c|c|c|c|c|c|c|c|c|}
\hline+ & 0 & - & 0 & + & - & + & - & 0,17 \\
\hline+ & & & 0 & & & & & 0,06 \\
\hline+++ & & & & & & & & 0,11 \\
\hline+ & & & 0 & & & & 0 & 0,09 \\
\hline+ & 0 & & 0 & 0 & - & & 0 & 0,10 \\
\hline+ & & & & & & & & 0,06 \\
\hline+ & & & & & & & & 0,06 \\
\hline+ & 0 & & 0 & 0 & - & & 0 & 0,08 \\
\hline
\end{tabular}

Du groupe 2

Hypochoeris achyrophorus L

Trifolium arvense $L$

Trifolium nigrescens Viv

Trifolium cherleri $\mathrm{L}$

Ornithopus compressus $L$

Calamintha nepeta (L) Savi

Erodium moschatum (L) L'Her

Foeniculum vulgare Miller

Filago pyramidata $\mathrm{L}$

Tuberaria guttata (L) Fourr

Helichrysum italicum (Roth) G Donfil

Anthemis arvensis $\mathrm{L}$

Medicago praecox DC

Paronychia argentea (L) Lam

Poa bulbosa L

Salvia verbenaca $\mathrm{L}$

Sedum caespitosum (Cav) DC

Spergula arvensis $\mathrm{L}$

Teucrium marum $\mathrm{L}$

Trifolium scabrum $\mathrm{L}$

Dianthus armeria $\mathrm{L}$

Trifolium tomentosum $\mathrm{L}$

Vulpia myuros (L) C C Gmelin

Du groupe 3

Carlina corymbosa L

Lathyrus cicera L

Anthoxanthum odoratum $\mathrm{L}$

Sanguisorba minor Scop

Sherardia arvensis $\mathrm{L}$

Aira caryophyllea L

Brachypodium ramosum (L) Roem et $S$

Cardamine hirsuta L

Cistus monspelliensis $\mathrm{L}$

Dactylis glomerata $\mathrm{L}$

Daphne gnidium $\mathrm{L}$

Erigeron canadense $\mathrm{L}$

Eryngium campestre $\mathrm{L}$

Euphorbia exigua L

Euphorbia peplus L

Fumaria officinalis $\mathrm{L}$

Lathyrus angulatus $\mathrm{L}$

Lotus parviflorus Desf

Medicago orbicularis (L) Bartal

Ononis spinosa $\mathrm{L}$

Pulicaria odora (L) Reichenb

Quercus ilex L

Santolina chamaecyparissus $\mathrm{L}$

Teucrium chamaedrys $\mathrm{L}$

Urospermum dalechampsi $(L)$ Scop ex Schmidt Vicia disperma DC

\begin{tabular}{|c|c|c|c|c|c|c|c|c|}
\hline & ++ & + & 0 & & & & & 0,14 \\
\hline & + & + & 0 & 0 & & & 0 & 0,07 \\
\hline \multirow[t]{2}{*}{-} & + & & + & 0 & - & 0 & 0 & 0,12 \\
\hline & + & & 0 & & & & + & 0,09 \\
\hline \multirow[t]{10}{*}{0} & + & 0 & 0 & 0 & 0 & -- & 0 & 0,18 \\
\hline & ++ & & 0 & 0 & 0 & & 0 & 0,08 \\
\hline & + & & 0 & - & & & 0 & 0,14 \\
\hline & + & & & & & & & 0,06 \\
\hline & + & & & & & & & 0,08 \\
\hline & + & & 0 & & & & & 0,08 \\
\hline & + & & 0 & & & & & 0,09 \\
\hline & ++ & & 0 & - & - & & 0 & 0,13 \\
\hline & + & & & & & & & 0,08 \\
\hline & ++ & & & & & & & 0,08 \\
\hline \multirow[t]{9}{*}{0} & ++ & 0 & 0 & 0 & 0 & -- & 0 & 0,13 \\
\hline & +++ & & 0 & & & & & 0,10 \\
\hline & + & & & & & & & 0,06 \\
\hline & + & & & & & & & 0,07 \\
\hline & + & & & & & & & 0,06 \\
\hline & ++ & & 0 & 0 & & & 0 & 0,11 \\
\hline & ++ & & 0 & & & & 0 & 0,12 \\
\hline & + & & 0 & & & & & 0,06 \\
\hline & + & & 0 & 0 & 0 & --- & 0 & 0,21 \\
\hline+ & 0 & +++ & 0 & 0 & 0 & - & 0 & 0,20 \\
\hline+ & & +++ & & & & & & 0,17 \\
\hline 0 & 0 & + & 0 & + & 0 & - & 0 & 0,10 \\
\hline 0 & 0 & + & 0 & + & 0 & - & 0 & 0,13 \\
\hline \multirow[t]{5}{*}{0} & 0 & + & 0 & 0 & - & --- & + & 0,15 \\
\hline & 0 & +++ & 0 & 0 & - & & 0 & 0,16 \\
\hline & & +++ & 0 & & & & 0 & 0,16 \\
\hline & & + & & & & & & 0,06 \\
\hline & & +++ & 0 & & & & 0 & 0,14 \\
\hline \multirow[t]{15}{*}{0} & 0 & + & - & 0 & 0 & - & 0 & 0,14 \\
\hline & & + & & & & & & 0,06 \\
\hline & & ++ & & & & & & 0,09 \\
\hline & & ++ & 0 & 0 & 0 & & 0 & 0,11 \\
\hline & & + & 0 & & & & & 0,13 \\
\hline & & + & & & & & & 0,06 \\
\hline & & + & & & & & & 0,07 \\
\hline & & ++ & & & & & & 0,06 \\
\hline & & + & & & & & & 0,06 \\
\hline & & + & & & & & & 0,06 \\
\hline & & ++ & & & & & & 0,09 \\
\hline & & + & 0 & 0 & & & 0 & 0,09 \\
\hline & & ++ & & & & & & 0,06 \\
\hline & & + & & & & & & 0,06 \\
\hline & & ++ & 0 & & & & & 0,08 \\
\hline \multirow[t]{2}{*}{0} & 0 & ++ & 0 & 0 & 0 & - & 0 & 0,13 \\
\hline & & ++ & & & & & & 0,09 \\
\hline
\end{tabular}


Tableau III suite.

Espèces différentielles 12 3 4
4 8 Inf mut

Du groupe 4

Bellis perennis $\mathrm{L}$

Erodium chium (L) Willd

Trifolium subterraneum $\mathrm{L}$

Capsella bursa pastoris (L) Medicus

Crepis bellidifolia Loisel

Geranium molle L

Lolium perenne $\mathrm{L}$

Medicago minima (L) Bartal

Poa annua $L$

Romulea ramiflora Ten

\begin{tabular}{|c|c|c|c|c|c|c|c|c|}
\hline-- & - & 0 & + & 0 & + & 0 & 0 & $\begin{array}{l}0,15 \\
0,12\end{array}$ \\
\hline & 0 & & ++ & 0 & -- & & 0 & 0,14 \\
\hline 0 & 0 & & $\begin{array}{l}+ \\
+\end{array}$ & - & 0 & - & 0 & $\begin{array}{l}0,11 \\
0,06\end{array}$ \\
\hline 0 & 0 & 0 & + & 0 & 0 & -- & 0 & 0,10 \\
\hline- & $\begin{array}{l}0 \\
0\end{array}$ & - & $\begin{array}{c}+++ \\
++ \\
+\end{array}$ & $\begin{array}{c}- \\
- \\
0\end{array}$ & $\begin{array}{l}0 \\
0 \\
0\end{array}$ & 0 & $\begin{array}{l}0 \\
0\end{array}$ & $\begin{array}{l}0,23 \\
0,08 \\
0,09 \\
0,06\end{array}$ \\
\hline 0 & 0 & 0 & $\begin{array}{l}0 \\
- \\
0 \\
0\end{array}$ & $\begin{array}{l}+ \\
+ \\
+ \\
+ \\
++\end{array}$ & 0 & 0 & 0 & $\begin{array}{l}0,09 \\
0,13 \\
0,06 \\
0,07 \\
0,10\end{array}$ \\
\hline-- & 0 & 0 & $\begin{array}{l}0 \\
0 \\
0 \\
0\end{array}$ & $\begin{array}{c}++ \\
+ \\
+ \\
+ \\
++\end{array}$ & 0 & - & 0 & $\begin{array}{l}0,16 \\
0,08 \\
0,09 \\
0,08 \\
0,06\end{array}$ \\
\hline
\end{tabular}

Du groupe 5

Bromus hordaceus L

Vicia sativa $L$

Avena barbata Pott ex Link

Bellis annua $\mathrm{L}$

Bromus sterilis L

Hypochoeris glabra $\mathrm{L}$

Raphanus raphanistrum $\mathrm{L}$

Serapias lingua $L$

Trifolium micranthum Viv

Vulpia ligustica (AlI) Link

Du groupe 6

Plantago major L.

Poa trivialis $\mathrm{L}$

Potentilla reptans $\mathrm{L}$

Veronica persica Poiret

Cerastium glomeratum Thuil

Crataegus monogyna Jacq

Cynosurus cristatus L

Daucus carota L

Festuca arundinacea Schreb

Holcus lanatus L

Thrincia hirta Roth

Lotus corniculatus $\mathrm{L}$

Poa pratensis L

Taraxacum officinale Wiggers

Trifolium pratense $\mathrm{L}$

Trifolium repens $L$

Du groupe 7

Agrostis stolonifera $\mathrm{L}$

Gaudinia fragilis (L) Beauv

Mentha aquatica $L$

Mentha pulegium $\mathrm{L}$

Ranunculus repens $\mathrm{L}$

Rumex conglomeratus Murray

Trifolium fragiferum $\mathrm{L}$

\begin{tabular}{|c|c|c|c|c|c|c|c|c|}
\hline & & & & & + & + & & 0,10 \\
\hline & & & 0 & - & + & + & 0 & 0,14 \\
\hline- & 0 & & 0 & 0 & +++ & + & 0 & 0,19 \\
\hline & & & 0 & 0 & + & & 0 & 0,10 \\
\hline & & & & & + & & & 0,07 \\
\hline & & & & & + & & & 0,07 \\
\hline & 0 & & 0 & 0 & + & & 0 & 0,12 \\
\hline 0 & 0 & 0 & - & 0 & + & - & 0 & 0,11 \\
\hline & & & & & + & & & 0,06 \\
\hline & & & 0 & 0 & +++ & & 0 & 0,14 \\
\hline & & & & & + & & & 0,08 \\
\hline 0 & 0 & 0 & 0 & 0 & + & 0 & 0 & 0,07 \\
\hline 0 & - & - & 0 & 0 & +++ & 0 & 0 & 0,20 \\
\hline & 0 & & 0 & 0 & + & & 0 & 0,07 \\
\hline & 0 & & 0 & 0 & +++ & & 0 & 0,18 \\
\hline- & -- & 0 & 0 & 0 & ++ & 0 & 0 & 0,15 \\
\hline & & & 0 & 0 & & +++ & 0 & 0,16 \\
\hline & & & - & & & ++ & 0 & 0,09 \\
\hline & & & & & & +++ & & 0,11 \\
\hline & & & 0 & & & + & & 0,11 \\
\hline 0 & -- & 0 & 0 & 0 & 0 & ++ & 0 & 0,14 \\
\hline & & & & & & + & & 0,08 \\
\hline & & & & & & +++ & & 0,19 \\
\hline & & & 0 & & & & + & 0,09 \\
\hline & 0 & & 0 & 0 & 0 & & + & 0,11 \\
\hline 0 & 0 & 0 & 0 & - & 0 & 0 & + & 0,11 \\
\hline & & & & & & & + & 0,09 \\
\hline 0 & - & & 0 & 0 & - & 0 & + & 0,15 \\
\hline 0 & 0 & & 0 & 0 & 0 & 0 & + & 0,07 \\
\hline & & & & & & & + & 0,06 \\
\hline & & & & & & & ++ & 0,08 \\
\hline & & & 0 & & & & + & 0,11 \\
\hline 0 & 0 & - & 0 & 0 & 0 & 0 & + & 0,08 \\
\hline
\end{tabular}

Du groupe 8

Bunias erucago $\mathrm{L}$

Achillea ligustica All

Anagallis arvensis $\mathrm{L}$

Dianthus armeria $L$

Medicago arabica (L) Hudson

Muscari neglectum Guss

Pteridium aquilinum (L) Kuhn

Trifolium incarnatum $\mathrm{L}$

Trifolium striatum L

Veronica arvensis $\mathrm{L}$

$\longrightarrow$


Tableau III suite.

\begin{tabular}{|c|c|c|c|c|c|c|c|c|c|}
\hline Espèces différentielles & 1 & 2 & 3 & 4 & 5 & 6 & 7 & 8 & Inf mut \\
\hline \multicolumn{10}{|l|}{ Espèces ubiquistes } \\
\hline Muscari comosum (L) Miller & 0 & - & 0 & 0 & 0 & 0 & 0 & 0 & 0,09 \\
\hline Agrostis castellana Boiss et Reuter & 0 & 0 & 0 & 0 & 0 & 0 & - & 0 & 0,06 \\
\hline Carex distachya Desf & & 0 & & 0 & 0 & 0 & & 0 & 0,06 \\
\hline Cerastium pumilum Curtis & 0 & 0 & & 0 & - & 0 & 0 & 0 & 0,08 \\
\hline Lolium multiflorum Lam & 0 & 0 & & - & 0 & 0 & 0 & 0 & 0,06 \\
\hline Parentucellia latifolia (L) Carvel & 0 & 0 & 0 & 0 & 0 & 0 & 0 & 0 & 0,06 \\
\hline Silene gallica $\mathrm{L}$ & 0 & 0 & 0 & 0 & 0 & 0 & - & 0 & 0,07 \\
\hline Trifolium campestre Schreber & 0 & 0 & 0 & 0 & 0 & -- & 0 & 0 & 0,11 \\
\hline
\end{tabular}

Tableau IV. Ordination des espèces dominantes différentielles de la variable "groupe», classées sur les signes positifs de leur profil écologique indicé (inf mut = information mutuelle entre une espèce et la variable "groupe") (détail des notations voir texte chapitre méthode).

\begin{tabular}{|c|c|c|c|c|c|c|c|c|c|}
\hline Espèces / Groupes & 1 & 2 & 3 & 4 & 5 & 6 & 7 & 8 & Inf mut \\
\hline Dactylis glomerata & +++ & & & - & & +++ & & & 0,21 \\
\hline Medicago minima & & + & & & & & & & 0,06 \\
\hline Poa bulbosa & & +++ & & 0 & & & & & 0,17 \\
\hline Anthoxanthum odoratum & & & + & 0 & & & & & 0,06 \\
\hline Brachypodium ramosum & & & $+t$ & & & & & & 0,08 \\
\hline Crepis bellidifolia & & & & + & & & & & 0,03 \\
\hline Lolium perenne & & & & +++ & - & & & 0 & 0,19 \\
\hline Poa annua & & & & + & & & & & 0,06 \\
\hline Trifolium nigrescens & & & & + & & & & & 0,08 \\
\hline Vulpia myuros & 0 & 0 & 0 & -- & +++ & - & 0 & 0 & 0,18 \\
\hline $\begin{array}{l}\text { Acnillea ingustica } \\
\text { Bromus sterilis }\end{array}$ & & & & & $\begin{array}{l}+ \\
+\end{array}$ & & & & $\begin{array}{l}0,04 \\
0,04\end{array}$ \\
\hline Ornithopus compressus & & & & & + & & & & 0,04 \\
\hline Trifolium micranthum & & & & & + & & & & 0,05 \\
\hline Cynosurus cristatus & & & & & & + & & & 0,07 \\
\hline Holcus lanatus & & & & & & + & & & 0,05 \\
\hline Poa pratensis & & & & & & +++ & & & 0,22 \\
\hline Trifolium pratense & & & & & & + & & & 0,05 \\
\hline Trifolium repens & & & & 0 & & ++ & & & 0,11 \\
\hline Agrostis stolonifera & & & & & & & + & & 0,05 \\
\hline Poa trivialis & & & & & & & $+t$ & & 0,05 \\
\hline Potentilla reptans & & & & & & & + & & 0,06 \\
\hline Ranunculus repens & & & & & & & + & & 0,05 \\
\hline Trifolium fragiferum & & & & & & & +++ & & 0,11 \\
\hline Carex distachya & & & & & & & & + & 0,05 \\
\hline $\begin{array}{l}\text { Trifolium subterraneum } \\
\text { Bromus hordaceus }\end{array}$ & 0 & 0 & & $\begin{array}{l}0 \\
0\end{array}$ & $\begin{array}{l}0 \\
0\end{array}$ & $\begin{array}{l}0 \\
0\end{array}$ & - & $\begin{array}{l}0 \\
0\end{array}$ & $\begin{array}{l}0,09 \\
0,04\end{array}$ \\
\hline
\end{tabular}

formation xérophytique de transition, maintenue par la pression du feu et qui sans cela évoluerait très rapidement vers des formations pyrophytiques à Cistus monspeliensis dominant, très courantes en Corse. Brachypodium ramosum est très fréquent dans ces milieux et il rejette après le feu à la façon de beaucoup de ligneux (Tra- baud, 1981) ce qui peut expliquer son important recouvrement lorsqu'il est dominant (CSm $=29 \%$ ). II peut être rencontré en association avec Anthoxanthum odoratum ( $\mathrm{CSm}=20 \%$ ), Vulpia myuros $(\mathrm{CSm}=19 \%)$ ou avec de nombreux trèfles annuels comme espèces codominantes. 
Le groupe 4 : ces formations mésophiles soumises à une forte pression de pâturage sont caractérisées par Erodium chium, Crepis bellidifolia et Medicago minima qui sont les espèces les plus "fidèles" de ce groupe. Deux caractéristiques des Tuberiaretea guttatae, Trifolium subterraneum et Medicago minima, sont encore rencontrées dans ces formations. Mais les fréquences élevées et les dominances de Lolium perenne et de Poa annua, taxons associés à la classe des Molinio-Arrhenatheretea et plus précisément à l'alliance du Trifolio-Cynodontion (Braun-Blanquet et Bolos, 1965), ainsi que l'importance de Trifolium nigrescens lié à la classe des Brometalia rubentitectori montrent que ce groupe assez fréquent (22\% des relevés) rassemble des formations subnitrophiles à nitrophiles (Rivas Goday et Rivas Martinez, 1963). Lolium perenne est fréquemment dominant, et dans ce cas, il assure en moyenne $20 \%$ du recouvrement végétal. Peuvent lui être associés comme codominants : Trifolium nigrescens (CSm $=23 \%)$, Trifolium subterraneum $(\mathrm{CSm}=23 \%)$, Bellis perennis $(\mathrm{CSm}=15 \%)$, Crepis bellidifolia $(\mathrm{CSm}=15 \%)$ et Poa annua $(\mathrm{CSm}=14 \%)$ pour les taxons les plus fréquents.

Le groupe 5 : cet ensemble de pelouses généralement fauchées et parfois un peu fertilisées est caractérisé par des espèces «fidèles" comme Vicia sativa, Avena barbata et Vulpia ligustica. II présente encore des espèces caractéristiques des Tuberiaretea guttatae, Vulpia myuros et Hypochoeris glabra qui relèvent une certaine sécheresse temporaire de ces milieux. De plus, la présence de Bromus hordaceus et de Avena barbata, caractéristiques de l'ordre des Brometalia rubenti-tectori (Rivas Martinez et Izco, 1977), donne à ce groupement un caractère subnitrophile. Vulpia myuros est l'espèce dominante la plus caractéristique de ce groupe (forte information mutuelle, of tableau IV). Sa contribution spécifique moyenne est d'ailleurs élevée $(25 \%)$, c'est-à-dire que dans les relevés où elle apparaît dominante, son recouvrement peut atteindre le quart du recouvrement végétal total de la station. Bromus hordaceus $(\mathrm{CSm}=18 \%$ ) et Trifolium micranthum $(\mathrm{CSm}=16 \%$ ) sont les espèces le plus souvent associées à Vulpia myuros dans le cortège des 3 premières espèces dominantes.

Le groupe 6 (milieux frais soumis à une fertilisation azotée d'environ $50 \mathrm{U} / \mathrm{ha}$ ) peut être classé dans les Arrhenatheretea à cause des fortes présences de Trifolium repens, Trifolium pratense, Poa pratensis, Holcus lanatus et Potentilla reptans, caractéristiques des pâturages à vivaces, mésohygrophiles, de bonne valeur pastorale et que l'on retrouve dans toute la région atlanticoeuropéenne (Chapu, 1973). La dominance de
Cynosurus cristatus, plante indicatrice de l'alliance du Cynosurion cristati Tx, 1947, et celle de Plantago major, caractéristique des Plantaginetalia, révèlent un bon niveau trophique et hydrique allié à une pression notable de pâturage. Poa pratensis, avec une contribution spécifique de $17 \%$ en moyenne dans les relevés où il domine, est l'espèce la plus caractéristique de ce groupe. Ses codominantes les plus courantes sont Trifolium repens $(\mathrm{CSm}=22 \%)$, Holcus lanatus $(\mathrm{CSm}=21 \%)$ Dactylis glomerata $(\mathrm{CSm}=20 \%$, Trifolium pratense $(\mathrm{CSm}=14,5 \%)$ et Cynosurus cristatus (CSm $=13 \%$ ).

Le groupe 7 correspond aux stations humides. Les espèces les plus fidèles à ces formations sont : Mentha aquatica, Trifolium fragiferum et Rumex conglomeratus. Ce groupe présente des taxons relevant de la classe des Agrostietea stoloniferae Oberdorfer (Camiz et al, 1984) : Trifolium fragiferum, Agrostis stolonifera, Potentilla reptans et Mentha pulegium. Notons que, au contraire, Gaudinia fragilis est associé à la classe des Isoeto nanojuncetea $\mathrm{Br} \mathrm{Bl}$ et $\mathrm{Tx}, 1943$, qui rassemble les formations inondées une partie de l'année. De son côté, Mentha aquatica est un élément de la classe Plantaginetea majoris Tx, 1943 , et elle révèle des périodes d'engorgement $\mathrm{du}$ sol en eau. Les espèces codominantes les plus caractéristiques de ce groupe sont Trifolium fragiferum (CSm $=20,5 \%)$, Potentilla reptans ( $\mathrm{CSm}=20 \%)$, Ranunculus repens $(\mathrm{CSm}=19 \%)$, Agrostis stolonifera (CSm $=18 \%$ ) et Poa trivialis (CSM $=11 \%$ ).

Le groupe 8 : la composition floristique différentielle de ce groupement ne permet pas de mettre en évidence d'analogie phytosociologique claire. La présence de Pteridium aquilinum, Achillea ligustica, Anagallis arvensis, Medicago arabica et Veronica arvensis, montre que ce groupe correspond à des friches en milieux plutôt frais. Seul Carex distachya y apparaît significativement dominant, mais son information mutuelle «espèce-groupe» est faible $(0,05)$ et, de plus, cette espèce appartient aussi au groupe des espèces ubiquistes (tableau III). Ces milieux d'humidité moyenne, faiblement ou non pâturés et non soumis à gestion par l'homme, peuvent être comparés à des friches postculturales anciennes. Aucune pression marquante des facteurs biotiques et abiotiques ne permet d'orienter la flore dans une direction déterminée et univoque. Aussi nous pouvons en conclure que ce groupe ne correspond pas à un type homogène car aucune caractérisation floristique précise ne peut y être nettement dégagée.

Nous considérons donc que les 7 premiers groupes (comportant 110 relevés) présentent, au contraire du groupe 8 , des éléments floristiques 
homogènes qui nous permettent de les assimiler à des types. Leur importance relative est inégale, le tableau II en donnant une approximation.

Il est intéressant d'analyser brièvement leur répartition régionale puisque nous avons vu le caractère informatif très synthétique de la variable région.

\section{Répartition régionale des 7 types (tableau V, fig 1)}

- Le type 1 est rencontré essentiellement en zone littorale (îles Lavezzi en particulier), sur sols profonds et sableux. Ce sont des formations de superficie limitée, souvent éloignées de tout bâti-

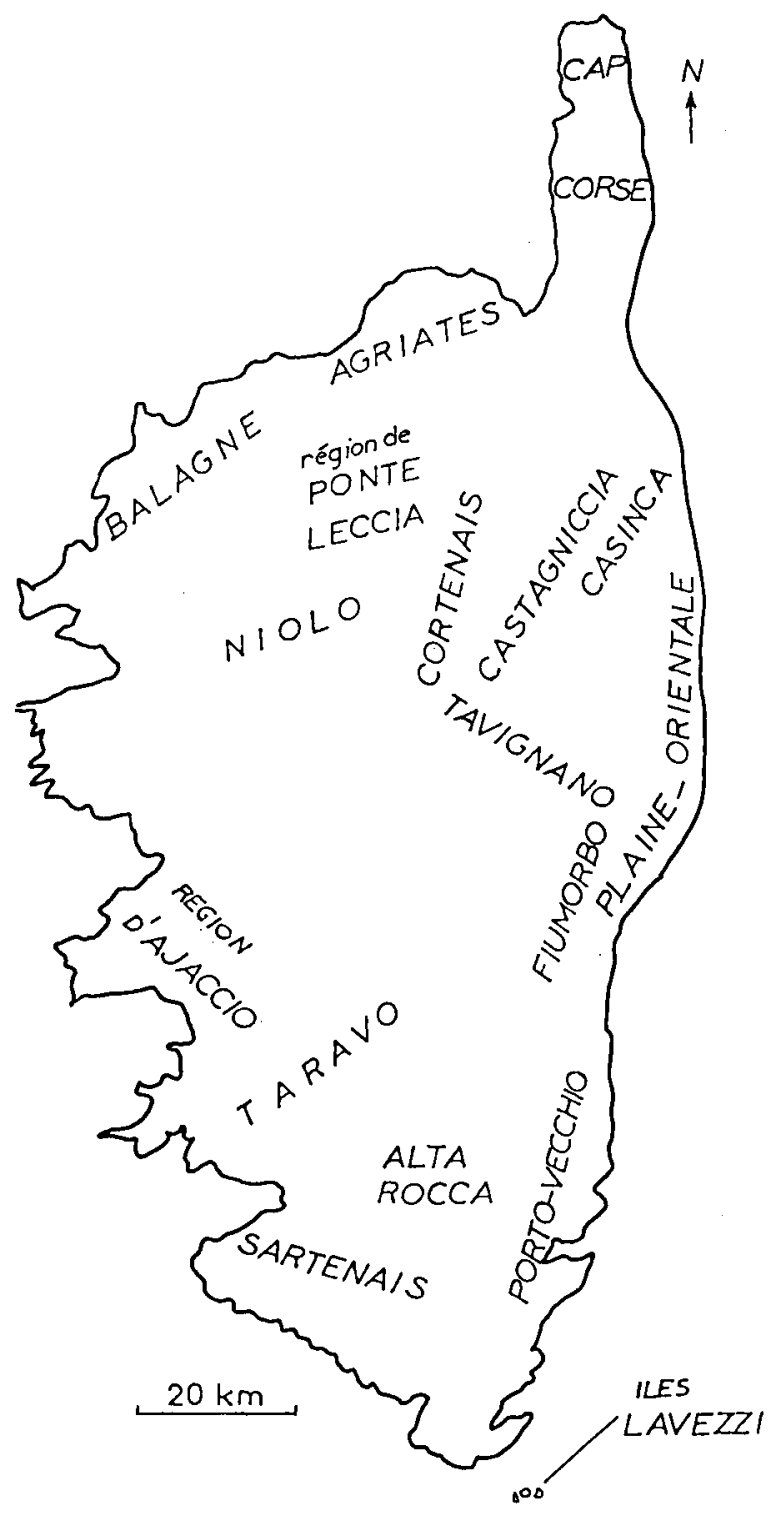

Fig. 1. Localisation schématique des principales régions naturelles de Corse échantillonnées dans notre étude. ment d'élevage, et où vaquent des troupeaux errants (bovins) à proximité des côtes.

- Le type 2, fréquent dans les régions de PonteLeccia et du Fiumorbo, se trouve sur pentes fortes en sols superficiels, avec de faibles recouvrements de végétation. Le type 2 et le type 4 (en situation de plus forte humidité, sur sols plus profonds), sont les types de milieu les plus fréquemment rencontrés sur les pelouses parcourues par les animaux, notamment par les ovins.

- Le type 3 , très révélateur de la région cortenaise et du Haut-Tavignano, sur roche mère schisteuse en mi-versants très pentus, se rencontre sur sols à faibles $\mathrm{pH}(6$ à 6,5$)$ avec une faible couverture végétale. Le feu, non contrôlé, ravageant chaque fois de nombreux hectares, ce type de milieu affecte de larges espaces qui correspondent, en général assez étroitement, à des parcours d'élevage.

- Le type 4 est souvent rencontré en Balagne, dans le cap Corse et beaucoup moins en Corse du Sud (ex Taravo) où les formations herbacées sont relativement plus entretenues et fertilisées. Le tapis végétal y est couvrant et les sols à dominante sableuse sont assez profonds. Nous avons montré (Volaire, 1984) la situation très caractéristique de ces pelouses dans le paysage corse : elles occupent des superficies de quelques dizaines ou centaines de $\mathrm{m}^{2}$ seulement, sur les replats, les crêtes, les cols ou dans des ouvertures de maquis. Elles servent de maillons aux trajets quotidiens observés par les troupeaux en libre parcours. Ce sont les lieux de prédilection des animaux qui s'y concentrent. Ainsi, ces taches d'herbacées, guettées par le maquis envahissant et agressif, n'existent que par l'effet de l'incessante pression de pâturage ; en effet, ces milieux sont en général éloignés des voies d'accès et non maîtrisés sur le plan foncier.

- Le type 5 est particulièrement rencontré en Plaine Orientale et dans la région du Bas et Moyen-Taravo. Ces prairies fauchées sont implantées sur sols profonds en mi-versants souvant bien exposés au sud et le recouvrement de leur tapis végétal est élevé.

- Le type 6 est relevé presque exclusivement dans certaines exploitations de l'Alta Rocca et du Haut-Taravo, soit à des altitudes élevées (600 à $800 \mathrm{~m}$ ) où les précipitations sont plus abondantes qu'en plaine. II s'agit de formations sur terrains plats à sol profond, ayant un pH supérieur à la neutralité. Ces types de milieu, en raison de leur gestion raisonnée (régime de pâturage tournant ou régime mixte pâture/fauche avec apports de fertilisants) peuvent être considérés comme des prairies à part entière. Elles sont, d'autre part, souvent situées près des bâtiments 


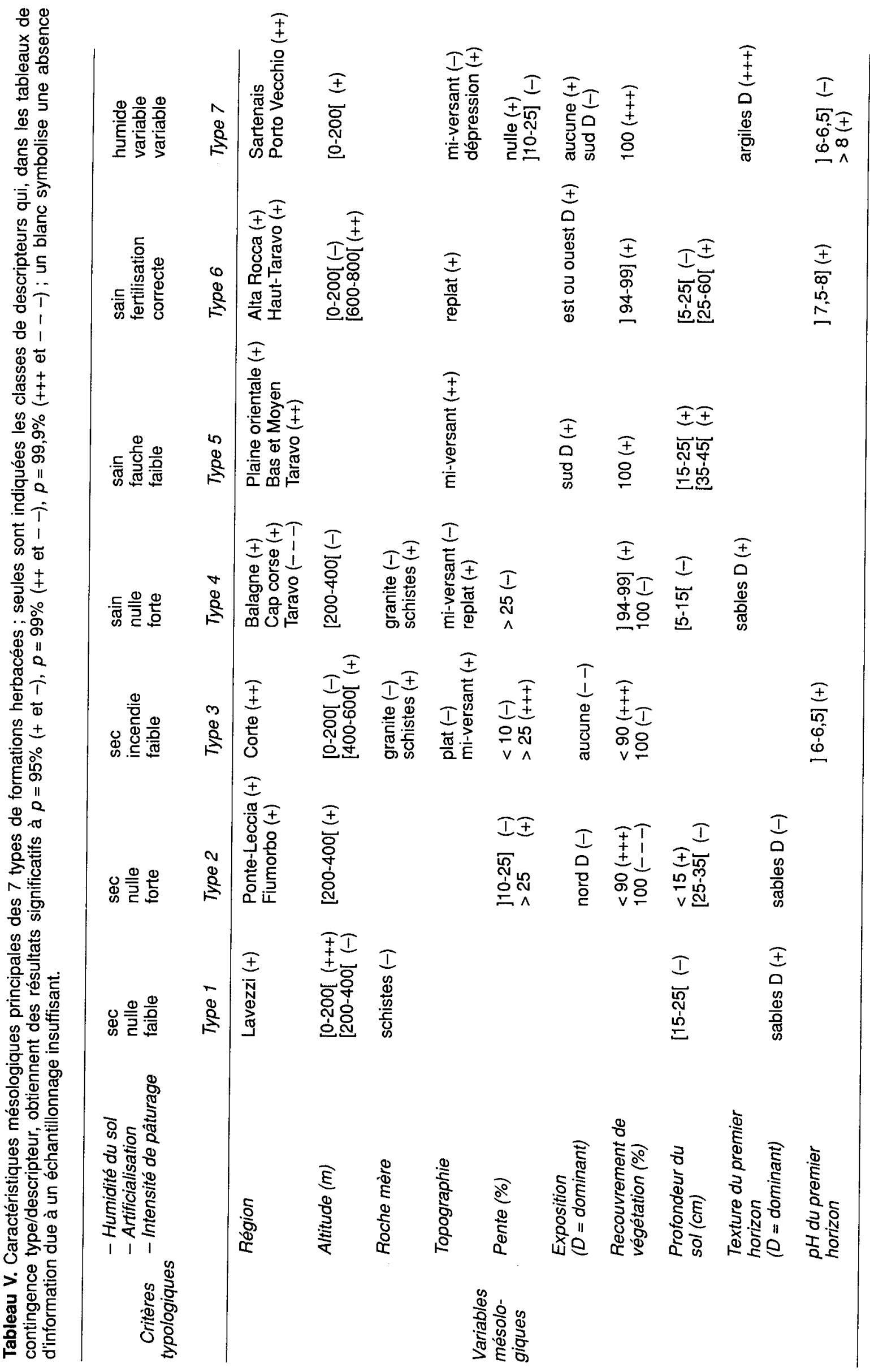


d'élevage, sur la surface principale que l'exploitant possède ou pour laquelle il dispose de la garantie d'un bail.

- Le type 7 est fréquent dans la région de PortoVecchio, donc à basse altitude et dans les basfonds. Les argiles dominent dans la texture du sol et le $\mathrm{pH}$ y est élevé (> 8). Le tapis végétal est continu et subit quelquefois de longues périodes d'engorgement en eau du sol.

\section{CONCLUSION}

Notre approche à l'échelle régionale essaie de rendre compte de la diversité rencontrée grâce à une grille simple et pratique d'interprétation des formations herbacées. Elle apparaît en conséquence relativement schématique puisqu'elle passe sous silence les milieux de transition et d'éventuels types peu représentés. Cependant les groupes d'espèces mis en évidence comme inféodés à tel ou tel type peuvent être apparentés à des groupes "imbriqués en écaille", plus ou moins superposés, qui expriment l'existence d'un continuum (résultant d'une variation progressive des milieux) plutôt que celle de strictes oppositions floristiques entre les types (Godron, 1967).

Par ailleurs, il est intéressant de souligner que, comme dans le cas de pâturages semi-arides (Rico Rodriguez et al, 1979), nos 7 types principaux se caractérisent bien sur la base de leurs seules espèces dominantes. Cet aspect apparaît utile pour le praticien pastoraliste, puisqu'il favorise l'identification rapide des formations herbacées étudiées et peut faciliter le diagnostic de leur intérêt fourrager à partir de la structure végétale des types auxquels on les rattache, question qui sera abordée dans un prochain article.

\section{REMERCIEMENTS}

Nous remercions vivement JB Casanova, agent pastoraliste au Parc naturel régional de Corse pour sa collaboration aux déterminations floristiques, ainsi que $M$ Roux, statisticien au CEPE-CNRS de Montpellier et J Gamisans, professeur à la faculté des sciences et techniques de Marseille, pour leurs suggestions et leurs critiques.

\section{RÉFÉRENCES}

Allier C, Lacoste A (1981) Processus dynamiques de reconstitution dans la série du Quercus ilex $L$ en Corse. Vegetatio 46, 83-91
Balent G, Duru M (1984) Influence des modes d'exploitation sur les caractéristiques de l'évolution des surfaces pastorales. Cas des Pyrénées centrales. Agronomie 4, 113-124

Benzecri JP (1973) L'analyse des données, 2 : l'analyse des correspondances. Dunod, Paris

Bobe V (1987) Étude phyto-écologique-agronomique et dynamique des prairies et des friches herbacées des valiées du Parc naturel régional de la haute vallée de Chevreuse. DAA INA-PG, $70 \mathrm{p}$

Bonin G, Aubert G, Barbero M, Gamisans J, Gruber M, Loisel R, Puezel P, Sandoz H, Thinon M, Vedrenne $G$ (1983) Mise en évidence de la dynamique de quelques écosystèmes forestiers et préforestiers provençaux aux étages méditerranéens SL à l'aide de taxons indicateurs. Vegetatio 54, 79-96

Braun-Blanquet J, De Bolos O (1956) Datas sobre las comunidades terofiticas de las llanuras del Ebro medio. Collectanea Botanica del Instituto Botanico de Barcelona 4 Fasc II (18), 235-242

Camiz S, Pignatti S, Ubrizsy A (1984) Numeral syntaxonomy of the class Agrostietea stoloniferae. Oberdorfer. Ann Bot Roma, 42, 135-147

Chapu A (1973) Les prairies de l'étage collinéen de la Franche-Comté centrale. Thèse d'Université, Besançon, $162 \mathrm{p}$

Daget $\mathrm{Ph}$, Poissonet J (1971) Une méthode d'analyse phytologique des prairies. Ann Agron 22 (1), 5-41

Daget P, Godron M (1982) Analyse de l'écologie des espèces dans les communautés. Masson, Paris, $163 p$

Delpech R (1975) Contribution à l'étude expérimentale de la dynamique de la végétation prairiale. Thèse de la faculté des sciences d'Orsay (Paris 1) $115 p+$ annexes

Etienne M (1977) Bases phyto-écologiques du développement des ressources pastorales en Corse. Thèse d'Université, Montpellier, $210 p$

Fournet J, Monestiez P (1987) Essai de caractérisation phyto-écologique des formations herbacées pâturées de Grande-Terre (Guadeloupe). Agronomie 7, 833-851

Gauthier B, Godron M, Hiernaux P, Lepart J (1977) Un type complémentaire de profil écologique : le profil écologique indicé. Can J Bot 55, 2 859-2 865

Gehu JM (1977) La végétation des pelouses sèches à thérophytes. In: Les pelouses sèches. Colloques phytosociologiques $n^{\circ} 6$. Lille $1977,12 p$

Godron M (1967) Les groupes écologiques imbriqués "en écailles". Oecol Plant 2, 217-226

Godron M, Daget P, Emberger L, Le Floch E, Poissonet J, Sauvage C, Wacquant JP (1968) Code pour le relevé méthodique de la végétation. CNRS Paris, $292 p$

Guckert A, Bonischot R (1984) The impact of climate on grass production and quality. Proceedings of the 10 th general meeting of the European grassland federation. Norway, 156-165

Hopkins A (1986) Botanical composition of permanent grassland in England and Wales. Relation to soil, environment and management factors. Grass Forage Sci 41, 237-246

Joffre R, Casanova JB (1983) Le développement des ressources fourragères des parcours en Corse de l'intérieur. Fourrages 93, 51-84 
Lepart J (1984) Intérêt et limites de l'analyse écologique au niveau régional. Les peuplements de chêne pubescent des hautes garrigues de Montpellier. Thèse de I'Université de Montpellier, $161 \mathrm{p}$

Loisel L (1976) La végétation méditerranéenne du Sud-Est continental français. Thèse ès-sciences, Université d'Aix-Marseille III, $384 \mathrm{p}$

Maranon-Arana T (1985) Floristic diversity and environmental heterogeneity in a "dehesa" of Sierra Morena. Ann Edaf Agrobiol 44, 1 183-1 197

Ozenda P (1964) Biogéographie végétale. Doin, Paris, $374 \mathrm{p}$

Pernet P, Lenclud G (1977) Bergers en Corse. Essai sur la question pastorale. Presses universitaires de Grenoble, $190 \mathrm{p}$

Rico Rodriguez M, Puerto Martin A, Gomez Gutierrez JM (1979) Tipificacion de pastizales semiaridos en funcion de sus especies dominantes. Pastos 9, 5-14

Rivas Goday S, Rivas Martinez S (1963) Estudio y classificacion de los pastizales espanoles. Ministerio de agricultura, Madrid, $270 p$

Rivas Martinez S (1977) Sur la syntaxonomie des pelouses thérophytiques de l'Europe occidentale. In: Les pelouses sèches. Colloques phytosociologiques $\mathrm{n}^{\circ} 6$ Lille, 55-71
Rivas Martinez S, Izco J (1977) Sobre la vegetacion terofitica subnitrofila mediterranea (Brometalia rubenti-tectori). Anal Inst Bot Cavanilles 34, 355387

Simi $P$ (1981) Précis de géographie physique, humaine, économique, régionale de la Corse. Collection Corse d'hier et de demain de la Société des Sciences historiques et naturelles de la Corse. $650 p$

Trabaud $L$ (1981) Man and fire: impacts on mediterranean vegetation. In: Mediterranean Type Shrubland. (Di Castri, eds), 523-536

Vacher $J$ (1984) Les pâturages de la Sierra Norte. Analyse phyto- et agro-écologique des dehesas pastorales de la Sierra Norte. Thèse d'Université, Montpellier, CNRS-CEPE Casa de Velàzquez Madrid, $195 \mathrm{p}$

Vallerand F (1985) Systèmes méditerranéens et utilisation des parcours. In: Systèmes d'alimentation des brebis laitières. Actes du $36^{\circ}$ congrès Fédération européenne de Zootechnie. Kallithea, Grèce, 237-251

Volaire F (1984) Utilisation du territoire dans trois élevages ovins corses. PNRC Mémoire ENITA, INRA, Corte $85 p$

Volaire F (1985) Approche méthodologique pour une étude des friches et pelouses de la Corse. Mémoire DEA, Université de Montpellier, $30 \mathrm{p}$ 